\title{
Pourquoi et comment favoriser la participation d'adolescents de ZUS à une recherche sur leurs mobilités urbaines?
}

Nicolas Oppenchaim

\section{(2) OpenEdition \\ Journals}

Édition électronique

URL : http://journals.openedition.org/cdg/2267

DOI : $10.4000 /$ cdg. 2267

ISSN : 2107-7266

Éditeur

UMR 245 - CESSMA

Référence électronique

Nicolas Oppenchaim, «Pourquoi et comment favoriser la participation d'adolescents de ZUS à une recherche sur leurs mobilités urbaines? 》, Carnets de géographes [En ligne], 3 | 2011, mis en ligne le 01 décembre 2011, consulté le 07 mai 2019. URL : http://journals.openedition.org/cdg/2267 ; DOI : $10.4000 /$ cdg. 2267

\section{(c) (i) () $\Theta$}

La revue Carnets de géographes est mise à disposition selon les termes de la Licence Creative Commons Attribution - Pas d'Utilisation Commerciale - Pas de Modification 4.0 International. 


\title{
Pourquoi et comment favoriser la participation d'adolescents de ZUS à une recherche sur leurs mobilités urbaines?
}

\author{
NICOLAS OPPENCHAIM \\ Observatoire du Samusocial de Paris \\ Laboratoire LVMT (Paris Est) \\ Sociologie \\ n.oppenchaim@samusocial-75.fr
}

\section{Résumé}

L'objectif de cet article est de présenter un exemple de participation active des adolescents à une recherche de sociologie sur leurs mobilités urbaines. Dans le cadre de cette recherche menée dans des établissements scolaires, les adolescents ont ainsi réalisé des questionnaires de sociologie qu'ils ont distribués à d'autres jeunes. Ils ont également pris des photographies et écrit des textes sur leurs mobilités, avant d'être interrogés individuellement sur ce thème par le chercheur. Cette méthode a pour premier avantage de favoriser le consentement éclairé des adolescents en leur faisant comprendre les implications de leur participation à une recherche. Elle permet également de créer une relation de confiance avec les adolescents et de stimuler leur réflexivité afin de les associer à l'élaboration de la recherche.

\section{Introduction}

Est-ce que prendre les adolescents comme objet d'étude suppose l'utilisation de méthodes de recherche différentes de celles utilisées pour les adultes ? Quelles sont les méthodes permettant de concilier rigueur scientifique et préoccupations déontologiques dans l'étude de cette population ? Ces questions ont été beaucoup plus abordées dans la littérature sociologique anglophone que dans celle en langue française (Danic et al, 2006). Or, elles se sont révélées centrales dans le cadre de la recherche que j'ai menée sur les mobilités quotidiennes des adolescents de zones urbaines sensibles (ZUS). Cette recherche avait pour point de départ l'idée que la mobilité constitue une étape importante de la socialisation des adolescents, car elle est le support du passage du monde familier au domaine public (Breviglieri, 2007). Elle permettait ainsi d'enrichir les approches statiques de la ségrégation, en ne résumant 
pas l'inscription urbaine des adolescents de ZUS à leur localisation résidentielle et en prenant en compte les interactions qu'ils ont avec des citadins d'une autre origine géographique et sociale durant leurs mobilités (Oppenchaim, 2009). Lors de cette recherche, j'ai alors été confronté à un certain nombre de difficultés méthodologiques et déontologiques, inextricablement liées: comment un enquêteur adulte peut-il accéder aux pratiques de mobilité des adolescents, qui sont un moment privilégié de l'entre-soi adolescent ? Comment recueillir et utiliser pour un travail académique des informations sur ces pratiques en s'assurant que les adolescents comprennent ce qu'implique leur participation à la recherche?

Ces différentes difficultés m'ont conduit à développer une méthodologie inédite dont le but était de favoriser la participation active des adolescents dans la recherche. J'ai ainsi mis en place des projets dans des établissements scolaires, combinant initiation des adolescents à la sociologie, réalisation de textes et de photographies sur leurs mobilités ainsi que des entretiens individuels semi-directifs. Afin de mieux comprendre la démarche méthodologique que j'ai suivie, je procéderai en trois temps. Je développerai tout d'abord les problèmes que soulève l'étude des pratiques de mobilité des adolescents. Puis, je montrerai en quoi la participation active des adolescents dans la recherche permet de résoudre en partie ces problèmes, avant d'exposer comment j'ai concrètement favorisé cette participation.

\section{Quels problèmes déontologiques et méthodologiques soulève l'étude des pratiques de mobilité des adolescents?}

La première difficulté méthodologique spécifique à laquelle est confronté un chercheur adulte travaillant sur les adolescents est de mener une recherche malgré la distance générationnelle qui existe entre lui et les enquêtés. En effet, les adolescents se situent dans une période de remise en cause du contrôle des adultes sur leurs pratiques et d'affranchissement vis-à-vis de la tutelle des institutions en charge de leur encadrement (Zaffran, 2010). Paradoxalement, la mise en relation du chercheur avec les enquêtés passe néanmoins majoritairement par ces institutions (école, centres sociaux, associations d'aide aux devoirs...), car les adolescents y passent une grande partie de leurs temps et que la présence d'adultes y est tolérée. Ce passage par les institutions ne concerne pas seulement les chercheurs s'intéressant aux pratiques des adolescents dans ces lieux, mais également ceux qui travaillent sur les pratiques se déroulant en dehors des cadres institutionnels. En effet, ces pratiques, comme les mobilités, constituent des moments privilégiés de l'entre-soi adolescent. Cela rend alors difficile la présence prolongée d'un enquêteur adulte auprès des adolescents lorsqu'ils réalisent ces activités. Le chercheur peut certes prendre comme terrain d'observation les lieux non institutionnels dans lesquels les adolescents se rendent durant leur temps libre, comme les centres commerciaux (Kokoreff, 1998). Néanmoins, les entretiens avec les adolescents constituent la source d'accès à ces pratiques la plus souvent utilisée. L'étude des pratiques extra-institutionnelles des adolescents est alors marquée par ce paradoxe: les institutions constituent une voie d'entrée privilégiée, voire unique, pour le chercheur, alors même que les adolescents souhaitent s'émanciper de la tutelle de ces institutions et éprouvent, pour certains, une relative méfiance à leur égard. 
Cette médiation par les institutions doit alors impérativement être intégrée à l'analyse des résultats obtenus. Elle n'est en effet pas sans influence sur la relation d'enquête entre des adolescents et un enquêteur adulte, plus ou moins assimilé à l'institution par laquelle il est entré en contact avec eux. L'enquêteur ne peut alors totalement s'émanciper des relations asymétriques, notamment en terme de pouvoir et d'autorité, qui structurent la relation de l'adolescent à cette institution. De même, cette médiation influence le profil des adolescents auxquels l'enquêteur accède. L'institution par laquelle le chercheur entre en relation avec les adolescents peut ainsi être en charge d'un public spécifique. Le choix des adolescents retenus pour l'enquête peut être également fortement orienté par les personnels institutionnels assurant I'interface avec le chercheur (Sime, 2008).

Le passage par des institutions pour accéder aux adolescents enquêtés pose donc un certain nombre de problèmes méthodologiques. Mais il soulève également des considérations éthiques, notamment en ce qui concerne le consentement éclairé à participer à la recherche. Par exemple, lorsque la mise en contact du chercheur avec les adolescents se fait par l'intermédiaire de l'institution scolaire, il peut exister une confusion aux yeux des adolescents entre la recherche proprement dite et les activités scolaires habituelles. La participation à la recherche pouvant alors être perçue comme obligatoire, l'adolescent risque de ne pas oser refuser la proposition. Ce problème du consentement éclairé ne concerne pas que les enquêtés mineurs (Vassy et Keller, 2008), mais il se pose de manière spécifique pour les adolescents pour trois raisons (Morrow, 2008). Ils bénéficient tout d'abord d'un statut juridique particulier, rendant nécessaire au niveau légal l'obtention d'une signature de leurs tuteurs adultes. Le simple accord des enquêtés mineurs n'est ainsi pas nécessairement suffisant pour couvrir le chercheur au niveau légal. D'autre part, les adolescents forment un groupe social plus vulnérable que les adultes. Au niveau individuel, ils peuvent se voir imposés lors de leurs interactions avec le chercheur les schémas d'interprétation de celui-ci, par manque d'habitude de ces situations. Au niveau collectif, ils ne possèdent pas de représentants dans la communauté adulte leur permettant de discuter les résultats tirés à leur égard ou de s'assurer que leur parole n'a pas été travestie ou retranscrite partiellement. Enfin, le consentement des adolescents à participer à la recherche peut être influencé par différents biais, comme par exemple la confusion évoquée précédemment entre activités obligatoires et activités de recherche lorsque celle-ci a lieu dans un cadre institutionnel.

La vulnérabilité potentielle des adolescents nécessite donc des précautions déontologiques, afin de s'assurer que les enquêtés comprennent les implications de leur participation à une recherche sociologique. Elle ne doit cependant pas conduire à ne pas investiguer cette population. Les considérations exposées précédemment sont ainsi assez analogues aux réflexions qui ont pu être menées dans le cadre d'enquêtes avec des groupes sociaux adultes vulnérables, comme les sans-domicile (Firdion et al, 1995). Les trois grandes justifications avancées lors de la mise en place du programme de recherche de I'INED sur cette population peuvent ainsi être transposées au cas des adolescents. Au niveau scientifique, ne pas mener d'enquête auprès d'adolescents reviendrait à se contenter du regard porté par les adultes sur leurs pratiques. Le chercheur n'accéderait pas à leur point de vue, mais à celui des institutions qui en sont en charge. Au niveau démocratique, ne pas enquêter sur les adolescents reviendrait à 
ne pas leur donner de statut de personne, à supposer une discontinuité entre leur monde et celui des adultes et donc à les exclure symboliquement de la société. Enfin, au niveau humain, les adolescents ne doivent pas être considérés uniquement sous l'angle de leur vulnérabilité. Ils peuvent ainsi avoir conscience de la forme de don/contre don impliqué par une situation d'enquête (Skelton, 2008). De même, ils peuvent avoir du plaisir à livrer leur point de vue à un adulte et à réfléchir sur leurs pratiques.

Au final, le positionnement éthique du chercheur est fortement influencé par le regard général qu'il porte sur l'enfance et l'adolescence (Morrow, ibid). Si l'adolescent est considéré uniquement sous l'angle de sa vulnérabilité, il n'est pas perçu comme compétent pour déterminer l'influence, positive ou négative, qu'aura pour lui la participation à une recherche. Au niveau déontologique, l'important pour le chercheur est alors d'obtenir une autorisation d'enquêter de la part des parents ou des institutions en charge de ces adolescents. D'autres chercheurs postulent au contraire que les mineurs possèdent les compétences pour comprendre les tenants et aboutissants d'une recherche et peuvent donc accepter ou refuser d'eux-mêmes leur participation (Masson, 2004 ; Skelton, ibid). Si le chercheur doit s'assurer au maximum qu'ils comprennent les conséquences de cette participation à court, moyen et long terme, le consentement des adolescents prime sur celui de leurs parents ou des institutions qui en ont la charge. Le chercheur ne peut donc se contenter de l'autorisation écrite des parents ou des personnels institutionnels. Au contraire, il doit faire primer le droit des enfants à s'exprimer plutôt que sa propre protection juridique vis-à-vis d'autres adultes. Par exemple, lorsque les adolescents sont en mesure de comprendre l'impact de l'enquête sur leur vie, il est parfois plus éthique d'agir en fonction du souhait de l'adolescent de donner son point de vue plutôt que de solliciter l'accord de parents qui pourraient s'y opposer :

"Where the child consents to participate the parent's consent is not required (...) where children can understand enough to distinguish research from other interventions and to understand the impact on them on participating, it may be more ethical to act on their consent than to require the fully informed consent of a parent. Such an approach gives children the maximum opportunity to have their views and experiences recorded and avoid the risk of exclusion of children whose parents would not respond to a request or would wish to control whom their child speaks to ${ }^{1}$ " (Masson, ibid).

Cette position est en adéquation avec la Convention de 1989 de l'Organisation des Nations Unies sur les droits de l'enfant, dont les articles 12 et 13 mettent en avant non seulement le droit de regard des enfants sur ce qui les concerne, mais également celui d'exprimer leur point de vue s'ils le désirent (Bell, 2008). Cette position commence peu

\footnotetext{
${ }^{1}$ Traduction de l'auteur: "Quand l'enfant consent à participer à la recherche, le consentement des parents n'est pas requis dans toutes les situations (...) quand les enfants sont assez informés pour distinguer l'activité de recherche d'autres interventions et pour comprendre l'impact qu'aura la participation à la recherche sur leur vie, il est parfois plus éthique de se baser sur leur consentement éclairé plutôt que de chercher à obtenir le consentement écrit de leurs parents. Une telle approche offre le plus d'opportunités aux enfants d'exprimer leur point de vue et leurs expériences. Elle évite également d'exclure des enfants, dont les parents ne répondraient pas aux demandes de consentement ou qui voudraient contrôler les interlocuteurs de leurs enfants".
} 
à peu à se diffuser dans le champ des études françaises (Danic et al, 2006). Elle est beaucoup plus répandue parmi les recherches anglo-saxonnes sur les pratiques sociales des adolescents, notamment celles qui sont publiées dans la revue Children's Geography et/ou celles qui s'inspirent de la "Participatory Action Research » (Hart, 1992). Dans cette méthode participative, les enquêtés participent activement au processus de recherche, ils identifient avec le chercheur les problèmes de leur communauté, ils choisissent les outils permettant de mieux comprendre ces problèmes et ils trouvent ensemble des solutions pour changer leur situation.

Nous avons donc vu que prendre les adolescents comme objet de recherche posait un certain nombre de problèmes méthodologiques (comment accéder aux pratiques qui se déroulent en dehors des cadres institutionnels) et déontologiques (comment faire comprendre aux adolescents ce que signifie de participer à une recherche, afin qu'ils puissent consentir, ou non, à y participer). Nous allons maintenant voir que la participation active des adolescents à la recherche permet de résoudre en partie ces différents problèmes.

\section{Quels sont les intérêts d'une participation active des adolescents à la recherche ?}

Les ouvrages ou revues de langue anglaise cités précédemment contiennent de nombreuses pistes permettant de favoriser le consentement éclairé des adolescents. La principale innovation méthodologique proposée est alors d'encourager la participation active des adolescents enquêtés dans la recherche. Cette participation est plus ou moins importante selon les recherches (Hart, ibid). Dans sa forme la plus simple, elle passe par exemple par la prise de photographies, la réalisation de cartes mentales ou la rédaction de textes par les enquêtés. La participation est plus importante lorsqu'un chercheur définit un thème général de recherche avant de commencer son enquête, mais qu'il implique ensuite les adolescents dans la construction des questions de recherche (Fine et al, 2003), qu'ils les laissent juges du choix de la méthode la plus adéquate à l'expression de leur point de vue (Skelton, ibid) ou qu'il les forme au recueil de données auprès d'autres jeunes (Alderson, 1995). Plus largement, des adolescents peuvent également participer à la définition des objectifs de la recherche en cours et faire partie de son comité de pilotage (Hart, ibid) alors que certains chercheurs défendent même l'idée d'une participation d'adolescents aux comités d'éthique des universités à chaque évaluation de projet incluant des enquêtés mineurs (Sime, ibid).

Cette participation active des adolescents comporte de nombreux avantages éthiques et scientifiques. La prise de photographies permet ainsi par exemple tout d'abord d'obtenir des informations sur des pratiques et des lieux non accessibles à un enquêteur adulte. Elle offre également l'avantage d'intégrer à la recherche des adolescents éprouvant des difficultés de verbalisation. Cette participation comporte aussi un aspect ludique, permettant d'entraîner l'adhésion d'adolescents ne souhaitant initialement pas se prêter au jeu de l'entretien ou de l'observation. Mais elle offre également d'autres avantages, notamment celui d'infléchir les problématiques de recherche du sociologue tout au long de l'enquête en y intégrant les capacités réflexives des adolescents (Sime, ibid). Les adolescents ne sont en effet 
pas des "idiots culturels", sans aucun regard réflexif sur leurs pratiques (Garfinkel, 1967). Cette participation active favorise également la compréhension des adolescents sur les enjeux éthiques d'une enquête sociologique, et éclaire en conséquence leur consentement à participer à la recherche.

Dans le champ français, ces méthodes de participation active des adolescents à la recherche ont été, à ma connaissance, mises en œuvre dans peu de travaux (Dubet et Martucelli, 1996; Lepoutre, 2005) ${ }^{2}$. Ces travaux mettent en évidence d'autres avantages de cette participation que ceux énoncés précédemment. Ils montrent tout d'abord comment il est possible d'articuler objectifs pédagogiques et production de connaissances scientifiques dans le cadre de projets menés dans des établissements scolaires (Lepoutre, ibid). La recherche ne conduit alors pas seulement à la reconnaissance du travail du chercheur par ses pairs, mais donne également naissance à un objet tangible (un livre, une exposition...) auquel les adolescents sont fiers d'avoir collaboré. Cela libère quelque part le chercheur de l'examen de conscience sur l'utilité de sa recherche pour les adolescents ayant accepté d'y participer. Ces travaux montrent également qu'il est nécessaire de laisser une place dans la recherche à la réflexivité des adolescents sur leurs pratiques. Le chercheur peut ainsi leur soumettre les interprétations qu'il a tirées à leur égard, afin d'en améliorer la pertinence (Dubet et Martucelli, ibid).

La participation des adolescents à la recherche favorise ainsi, entre autres, leur consentement éclairé, elle permet l'accès à des pratiques peu accessibles à un enquêteur adulte, elle peut entraîner l'adhésion de jeunes ne souhaitant pas initialement répondre à des questions, elle évite de recueillir des discours trop formatés par les propos que les adolescents ont l'habitude de tenir aux adultes... Une dernière considération générale explique l'intérêt que j'ai porté à ces méthodes. Elles sont en adéquation avec ma perspective théorique sur la mobilité des adolescents de ZUS. Dans leurs déplacements, ces adolescents sont confrontés à des situations problématiques, notamment dans leurs interactions avec des citadins dont ils ne sont pas familiers. Ces épreuves, même les plus minimes, peuvent alors conduire à un retour réflexif de l'adolescent sur ses habitudes d'action et à leur modification. Le retour des adolescents durant des entretiens sur les épreuves qu'ils ont rencontrées dans leur mobilité n'est alors possible que s'ils adoptent sur leurs pratiques un regard réflexif, ce qui est un des intérêts de leur implication active dans la recherche.

Pour conclure cette partie, soulignons que l'ensemble des considérations déontologiques soulevées jusqu'à présent ne sont pas totalement spécifiques aux adolescents. Tout chercheur qui étudie dans la durée un monde social est confronté à un moment ou un autre à ces considérations, notamment lorsqu'il travaille sur un monde social dominé (Lepoutre, ibid). N'est-il pas en train de trahir la confiance des enquêtés? Ne profite-il pas de leur confiance à des seuls fins de promotion académique, alors que le sort des enquêtés ne sera pas modifié par cette recherche ? Les enquêtés ont-ils conscience qu'une partie de leurs pratiques, même anonymisées, risquent d'être portées sur la place publique ? Comment déterminer les pratiques qu'il

\footnotetext{
${ }^{2}$ Ce recensement n'est pas exhaustif. Dans son livre, D. Lepoutre donne ainsi l'exemple de trois enseignants ayant mené des projets de recherche-action avec leurs élèves. Il cité également la thèse de H. Vieillard-Baron, dont un des volumes est constitué de récits de vie écrits par des lycéens.
} 
convient de révéler ou au contraire de laisser dans l'ombre pour ne pas nuire aux enquêtés? Là aussi, la compréhension par les enquêtés de l'implication de leur participation à la recherche est essentielle et elle ne peut pas se limiter à la signature d'un formulaire de consentement à participer. Les méthodes visant à favoriser la participation active des enquêtés afin de résoudre, en partie, ces problèmes déontologiques n'ont d'ailleurs pas seulement été mises en œuvre avec des mineurs. Citons par exemple, dans une perspective théorique proche de la mienne sur la mobilité, le projet qu'l. Joseph menait sur la ligne de métro 2 à Paris. Celui-ci souhaitait substituer à l'observation participante traditionnelle une " ethnographie participative " avec des itinéraires commentés d'usagers du métro, des autoconfrontations entre citadins ayant des conflits d'usage ainsi que des forums hybrides composés de gestionnaires et d'usagers (Tonnelat, Jolé et Kornblum, 2007).

\section{Des projets autour de la mobilité menés dans huit établissements scolaires}

Avant de présenter plus en détail les projets que j'ai menés dans des établissements scolaires, il convient de rappeler qu'ils ont suivi chronologiquement une ethnographie d'un an avec de jeunes garçons (13-18 ans) fréquentant la maison de quartier d'une ZUS de grande couronne. J'y ai été confronté aux difficultés habituelles rencontrées par un ethnographe dans son travail de terrain avec des populations défavorisées. II m'a fallu ainsi, classiquement, faire avec la distance sociale qui me séparait des jeunes afin d'acquérir un "savoir être avec " les adolescents. Cette distance sociale était également redoublée par une distance générationnelle avec les adolescents, qui ne me percevaient ni comme un animateur, ni comme un chercheur, mais me situaient quelque part entre ces deux professions. La présence quotidienne parmi ces jeunes, dans le quartier et dans les trains, l'accompagnement de sorties, la réalisation de vingt entretiens ethnographiques $\mathrm{m}^{\prime}$ ont alors permis de recueillir un riche matériau d'information sur les pratiques de mobilité des adolescents du quartier. Elle m'a aidé d'une part à mieux comprendre les interdépendances entre ancrage résidentiel et pratiques de mobilité des adolescents, mais également qu'une des principales épreuves que ces derniers affrontaient dans leurs mobilités était la confrontation aux autres citadins en raison du triple stigmate sociale, ethnique et générationnelle dont ils se sentent porteurs (Oppenchaim, 2011).

Je ressentais cependant une insatisfaction éthique durant cette ethnographie. En effet, si la plupart des jeunes acceptaient de me faire partager en partie leur quotidien et de répondre à mes questions, cela était le plus souvent beaucoup plus dû à une sympathie à mon égard qu'à une réelle compréhension des enjeux et intérêt de mon travail de recherche. Très peu d'adolescents comprenaient l'intérêt de se pencher sur leurs pratiques de mobilité, la plupart y voyant malgré leur sympathie une manière détournée des institutions de contrôler leurs moments de liberté hors du cadre des différentes institutions dans lesquelles ils sont insérés (école, travail social, police....). Cette absence de compréhension me questionnait alors sur le sens de la démarche sociologique, notamment "savoir pour qui on écrit et dans quel but ? " (Lepoutre, ibid). Cette question se pose généralement au moment de la restitution de la recherche et du recueil de gains symboliques de la part de l'enquêteur. Elle ne cessait cependant de me tarauder au moment de l'enquête, ayant l'impression de recevoir 
des histoires de vie singulière de la part de ces jeunes, sans rien leur apporter en retour. C'est alors cette insatisfaction qui a nourri mon intérêt pour les méthodes favorisant l'implication active des adolescents dans la recherche. Ces méthodes me semblaient d'autant plus intéressantes que certains adolescents de la maison de quartier développaient une vraie réflexion sur leurs pratiques de mobilité. Un d'entre eux m'expliqua ainsi un jour qu'il avait plus tendance à fréquenter Châtelet que les Champs Elysées, car malgré la présence massive de policiers il y était beaucoup moins contrôlé. Interrogé sur les raisons de ces contrôles plus nombreux aux Champs-Elysées, il les expliqua par la présence plus importante de touristes, définissant au contraire Châtelet comme un lieu de passage où la présence des jeunes était plus tolérée.

Or malgré tous mes efforts, il m’a été impossible dans le cadre de mon ethnographie de mettre en œuvre ces méthodes. Cela était sans doute dû à la spécificité de mon terrain d'étude, une maison de quartier considérée par les jeunes essentiellement comme un lieu de loisirs et de retrouvailles à l'écart des regards des personnes plus âgés du quartier. J'ai ainsi proposé à certains jeunes avec lesquels j'avais déjà réalisé un entretien classique de prendre des photographies durant leurs déplacements puis de les commenter. La plupart me déclaraient cependant avoir la "flemme " et que cela leur rappelait trop le cadre scolaire. Cette difficulté à mettre en œuvre ces méthodes était également renforcée par le turn-over des jeunes fréquentant la maison de quartier, qui pour certains ne venaient que pour une heure ou de manière espacée dans le temps.

J'ai alors complété cette ethnographie par des projets menés dans huit établissements scolaires (quatre classes de troisième, deux secondes professionnelles BEP vente et deux secondes générales). Ces projets articulaient trois dimensions : d'une part, une initiation des élèves à la sociologie, sous la forme de la réalisation et de la passation d'un questionnaire à d'autres adolescents ; d'autre part la réalisation de textes et de photographies autour de leur mobilité; enfin quatre-vingt quinze entretiens semidirectifs d'une heure, réalisés après l'initiation à la sociologie et donnant lieu dans la majorité des cas à une restitution collective de mon enquête devant l'ensemble des élèves.

Mener une recherche dans des établissements scolaires suppose tout d'abord de nouer une relation de confiance à la fois avec les élèves et avec les professeurs. La construction de cette relation dans le cadre scolaire ne va pas de soi, car un nombre important des adolescents de ZUS entretient un rapport conflictuel avec l'institution scolaire. Le principal biais que je devais éviter était d'être considéré par les élèves comme un professeur, ou du moins d'être assimilé à l'institution scolaire. Chaque prise de contact avec les élèves comprenait ainsi une présentation de la sociologie et de ma démarche de recherche, en précisant bien que je n'appartenais pas institutionnellement à l'établissement. J'ai alors cherché à casser le cadre scolaire de différentes manières : en évacuant l'attente de la note (c'était à chaque fois une des premières questions qui étaient posées par les élèves) ou par la possibilité de tutoiement et d'appellation par le prénom de manière réciproque. Le fait d'être un jeune chercheur ne partageant pas totalement les codes vestimentaires et de langage des professeurs a sans doute également participé à casser ce cadre scolaire : " tu n'as pas la voix clean comme un prof " me confia ainsi un jour un jeune lors d'un entretien. 
La familiarisation antérieure avec les codes, notamment de langage, des adolescents de ZUS lors de mon ethnographie m'a aussi sans doute aidé à ne pas être perçu comme appartenant à l'institution scolaire.

Casser ce cadre scolaire avait pour principal but de renforcer l'idée d'égalité dans la construction de la recherche, les élèves m'apportant autant que je pouvais leur apporter, en particulier un projet allant à l'encontre de la routine scolaire. J'étais ainsi sans doute pour les élèves un objet aussi étrange que j'avais pu l'être pour les adolescents de la maison de quartier: un intervenant extérieur qui n'est pas un professeur et n'en partage pas totalement les codes. Je ne dis pas que les élèves ne peuvent se confier aux professeurs, mais ne pas être identifié à une figure d'autorité m'a semblé faciliter l'implication des élèves. Il convient néanmoins de ne pas être naïf : comme nous le verrons ultérieurement, l'implication plus ou moins importante de certains élèves dans le processus de recherche, notamment dans l'élaboration de questionnaires, a pu être motivée en partie par la pression de leurs professeurs. Cette mise entre parenthèses temporaire du cadre scolaire lors de mes interventions était tolérée par les professeurs d'Histoire-géographie, de Français, de Vente ou d'Arts Plastiques qui avaient accepté de travailler avec moi. Ces derniers devaient combiner les intérêts de recherche du sociologue, l'adhésion des élèves, ainsi que leurs propres objectifs pédagogiques. Il s'agissait généralement, même si cela est difficilement quantifiable, de professeurs dynamiques, atypiques pour certains, mais qui partageaient un bon relationnel avec les élèves.

Au final, sans être totalement assimilé par les adolescents à l'institution scolaire, j'ai donc pu bénéficier des avantages que peut apporter un cadre scolaire par rapport à celui de la maison de quartier, notamment pouvoir mener des projets dans la durée en y impliquant activement les adolescents. Rappelons néanmoins qu'il existe des degrés dans la participation des adolescents dans la recherche. Celle-ci peut aller de l'information des enquêtés sur les objectifs et les conséquences de l'enquête au choix par les adolescents du sujet à investiguer (Hart, ibid). Or, les élèves n'ont pas participé directement au choix du thème général de ma recherche ou à celui des outils, même s'ils disposaient d'une grande marge dans les modalités concrètes d'utilisation de ces outils (thèmes à investiguer dans le questionnaire, liberté dans la forme d'écriture des textes, rendu des résultats sous la forme d'une exposition ou d'un blog). Sur l'échelle de participation des enfants à la recherche élaborée par R. Hart (1992: 8), je me situe donc au sixième échelon sur huit (le chercheur décide du thème général, mais discute avec les enfants des meilleurs moyens de la mener). L'imposition d'un thème général et la réalisation d'entretiens classiques à la fin des projets m'a cependant sans doute permis de ne pas être perçu par certains adolescents comme un simple animateur, ce qui a pu renforcer leur sérieux et leur implication dans les projets.

La première dimension de ces projets a été la réalisation de questionnaires par les élèves sur des thématiques propres à l'adolescence qu'ils avaient auparavant choisies : les relations amoureuses et amicales entre adolescents, le rapport des adolescents à leur quartier et à la ville en général, les adolescents et l'organisation de leur temps. Les élèves ont ensuite distribué ces questionnaires à d'autres adolescents avant qu'une restitution des résultats ne leur soit faite en classe entière. Cette initiation à la sociologie a présentée plusieurs avantages. D'une part, elle a permis de faire 
comprendre aux élèves les enjeux déontologiques et scientifiques d'une enquête. Ces derniers ont ainsi dû expliquer à d'autres adolescents, qu'ils n'avaient parfois jamais vus, que des réponses personnelles, par exemple sur leur sexualité, étaient anonymes et donneraient lieu à une restitution en classe, sans que leur nom soit révélé. Ils ont également dû faire comprendre à ces adolescents en quoi leurs réponses présentaient un intérêt de recherche. Les élèves ont ainsi pu saisir en pratique ce qu'impliquait de se prêter à une enquête sociologique.

Cette initiation offrait également des avantages sur le plan scientifique. Si les thèmes des questionnaires étaient divers, ils posaient en filigrane des questions propres à ma recherche, en particulier les disparités entre filles et garçons sur les horaires de sortie. La restitution des résultats des questionnaires en classe entière a ainsi donné lieu à des discussions très fournies et a obligé les élèves à réfléchir et à argumenter sur certaines spécificités de leurs pratiques. Elle a également permis de libérer la parole de certains jeunes et de préparer ainsi les entretiens individuels qui ont suivi. Le côté ludique de la réalisation et de la passation des questionnaires a également pu contribuer à créer une relation de confiance avec les adolescents et à favoriser leur participation ultérieure à ces entretiens.

Enfin, dans certains projets il a été décidé que les élèves ne distribueraient pas seulement le questionnaire aux adolescents de leur quartier mais également de manière collective à la sortie de grands lycées du centre de Paris. Cela avait pour premier avantage de faire réfléchir les élèves sur les disparités entre adolescents banlieusards et parisiens. Plus largement, cela donnait l'occasion à certains élèves qui ne s'y étaient jamais rendus, de fréquenter les quartiers centraux de Paris. Ils étaient ainsi confrontés, lors de la distribution en tête à tête des questionnaires, à l'altérité d'adolescents d'un autre milieu social. Une partie des élèves se rendaient ainsi initialement à contrecœur à Paris, ayant peur que personne n'accepte de répondre à leurs questions. S'ils avaient au début faiblement confiance en eux, ils se sont rendus peu à peu compte qu'ils pouvaient réussir à obtenir l'attention des adolescents parisiens, le statut d'enquêteur permettant par ailleurs de suspendre le temps d'une interaction le stigmate social dont une partie se sentait porteuse. Lien de cause à effet ou non, ces élèves ont été ensuite beaucoup plus nombreux que la moyenne à effectuer leur stage professionnalisant dans Paris intra-muros. Le but premier de la recherche était la production de connaissance, et non de faire évoluer, même à la marge, les compétences de mobilité des élèves. Néanmoins, cette distribution du questionnaire dans Paris, ainsi que l'enthousiasme d'une majorité des élèves pour les projets, offrait également l'avantage d'apaiser mes interrogations sur l'utilité immédiate de ma recherche.

Parallèlement ou après cette initiation à la sociologie, les élèves menaient également des travaux d'écriture et photographique sur le thème de la ville et des mobilités. Ces travaux permettaient de préparer les entretiens ultérieurs en donnant un côté ludique à la recherche, en renforçant ou en créant une relation de confiance avec les adolescents et en favorisant le retour réflexif sur leurs pratiques. Ils donnaient également des informations directes sur les pratiques de mobilité de ces adolescents. La prise de photographies des élèves sur leurs mobilités a été faite selon deux grandes modalités, en raison de différentes contraintes financières. Lorsque j'ai réussi à obtenir 
des financements, du Conseil Départemental de Seine Saint Denis ou des classes APAC du rectorat, les élèves étaient accompagnés par un photographe professionnel durant une après-midi. En l'absence de financement, les élèves prenaient eux-mêmes des photographies sur leur quartier ou les lieux fréquentés durant leur mobilité, à l'aide d'appareils jetables distribués ou le plus souvent avec leur propre appareil ou téléphone portable. Ces photographies permettaient d'intégrer de manière ludique des adolescents pouvant avoir des difficultés ou une réticence initiales à verbaliser leurs pratiques. La présence du photographe professionnel présentait l'avantage supplémentaire de permettre une initiation à la photographie, ainsi qu'une familiarisation à certains lieux qu'ils ne connaissaient pas, les déplacements se faisant le plus souvent par groupe de trois. Au niveau scientifique, ces déplacements ont permis un retour réflexif des jeunes sur les lieux qu'ils fréquentent, ces derniers explicitant durant le trajet pourquoi ils choisissaient ce lieu, ce qu'il leur évoquait, pourquoi ils insistaient sur tel aspect dans leur prise de vue, etc. Ils me permettaient également de renforcer la relation de confiance avec les jeunes et de pouvoir ensuite faire un retour approfondi sur les lieux photographiés durant l'entretien individuel.

Figure 1 : Photographie et texte d'un élève de troisième générale (avril 2009)

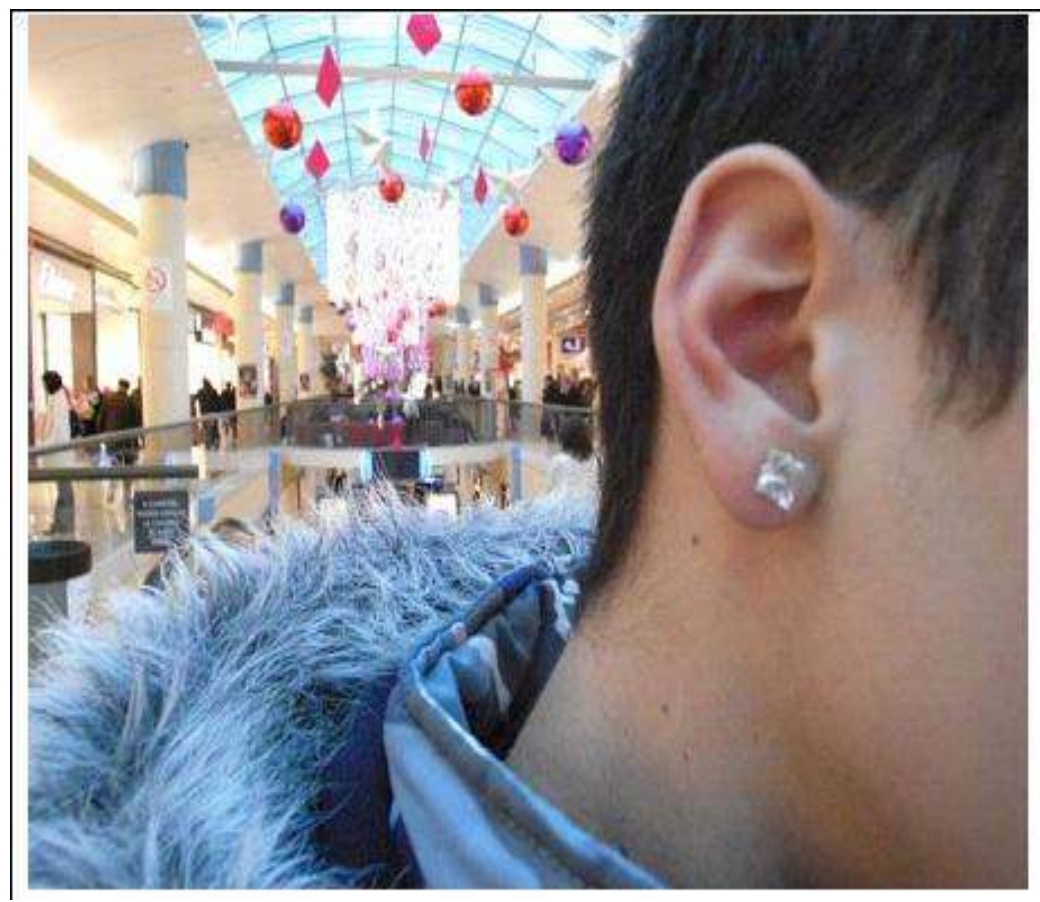
Rosny 2
Les week-ends, je sors avec mes potes, on joue au foot, ou alors on se livre à notre activité préférée: draguer les filles... Donc on va à Rosny 2 et puis au passage, on passe par Score Game.
On regarde les nouveautés et on va aussi à Micromania, on ne sait jamais et puis comme on aime beaucoup le McDo, on $y$ va faire un tour et puis on rentre.
Au final, on n'a pas trouvé de jeux, on a bien mangé et sur 4 approches, 3 râteaux, mais c'est pas grave...
On retentera notre chance le week-end prochain...

Ces prises de photographies ont été complétées par un travail d'écriture des élèves, soit directement à partir des clichés, soit en s'appuyant sur des descriptions urbaines d'écrivains. Je craignais que cette dimension du projet soit perçue comme trop scolaire, mais elle a recueilli l'adhésion de la majorité. Cela s'explique sans doute par la liberté de forme dont ils disposaient pour décrire leur quartier et leur rapport à la ville : écriture de slams, de poèmes, description neutre du quartier de résidence ou des lieux fréquentés en s'inspirant des œuvres de Georges Pérec dans Espèces d'espaces. J'ai choisi, en commun avec les professeurs de Français, ce livre comme support d'aide aux élèves pour décrire leur quartier, car il contient de nombreux passages qui 
fournissent un mode d'emploi des descriptions sociologiques sur la ville (Becker, 2010).

\section{Figure 2 : Texte d'un élève de seconde générale (mars 2009)}

Ma rue,..., je dois parler de ma rue... très bien. Comment ? Le plus platement possible, d'accord, j'me lance.

Tout d'abord je n'ai pas vraiment de rue. C'est plutôt un grand bâtiment donnant sur un parking. Sur ce parking de 34 places, une quarantaine de voitures sont stationnées. II est 19 heures, tout est normal comme d'habitude. Du haut de mes 15 étages je peux apercevoir une grosse flaque d'huile se déversant d'une voiture sans roue, les restes d'une voiture brûlée volontairement d'un acte criminel. De chez moi, je peux voir pratiquement tout le Bois Saint Denis, l'aéroport Charles de Gaulle et ses trois terminaux ses trois tours de contrôle et ses quelques 500 vols par jour, une bonne partie du centre-ville et son brouhaha, l'enseigne de "Leroy Merlin " de Livry Gargan clignotant toutes les trois secondes et demie, et le phare de Paris, l'œil de Paris brillant de mille feux à la tombée de la nuit, tournoyant et m'éblouissant à vingt quatre secondes d'intervalle.

En bas, devant mon hall, on peut entendre un jeune murmurer à un vieillard : "J'te donne une dix ». Le vieux répondit " Non, je, je...je veux une trente ! ". " Ah nan ici, c'est moi qui choisis, j'te passe une dix ! Un point c'est tout ! ». " Très bien tu as gagné, donne moi une ».

Ou bien un autre, encore plus jeune, essayant de suivre les paroles, incompréhensibles pour lui, d'un rappeur américain qu'il a soigneusement téléchargées illégalement et mis sur son IPod vidéo troisième génération huit gigas, qu'il a volé à un pauvre homme dans le RER (il s'en vante tous les jours).

Sur le mur qui fait face au parking, on remarque que le numéro de mon immeuble est le quatre-vingt treize ou plutôt le trois avec, peint à la peinture blanche, un neuf devant, pour rendre le bâtiment plus beau. C'est bien, c'est créatif, comme quoi avec peu on peut faire beaucoup.

Sur le mur de la gauche, un petit dégradé de couleurs qui devient de plus en plus foncé. C'est le jeune qui écoutait du rap « US » qui vient d'arriver ici. II n'habite pas dans ce bâtiment, mais il y reste jour et nuit et il n'a aucune honte ou pudeur.

Ah! Je viens de voir un petit garçon seul qui a failli se faire renverser par une Renault 25 qui voulait se garer. Le petit courait derrière son ballon Némo en pleurant car il avait été percé par le chien d'un jeune qui, faisant des tractions à l'arrêt de bus frime avec ses muscles et ses deux bébés Rottweiler. Les deux Rottweilers ont les oreilles et la queue coupées aux ciseaux par leur maître, car comme ces chiens font des combats, il vaut mieux qu'elles soient coupées, car si, lors d'un combat, l'un d'eux perd une oreille, ce pourrait être très embêtant et humiliant pour le maître.

Une bande d'adolescents, sûrement collégiens, avec leurs cartables imbibés de Tipex, viennent de passer dire bonjour aux jeunes postés devant mon bâtiment, ils prennent sur eux alors qu'il ne le faut pas! Après les avoir salués, ils reprennent leur balade avec ce qu'ils appellent une dégaine. J'appelle ça boiter (mais c'est leur choix). Ils rencontrent deux jeunes demoiselles "fashion ", les adolescents se ruent sur elles comme s'ils avaient aperçus leur idole. Certains leur font la bise et d'autres leur serrent la main pour montrer leur indifférence. Ils partent ensemble sur le côté du bâtiment que je ne peux pas observer.

Je ferme la fenêtre de ma cuisine d'où je vous décris mon environnement quotidien, grâce à des jumelles que j'ai utilisées pour plus de précision. La fermeture de cette fenêtre permet une coupure entre le bruit assourdissant des klaxons du 619 et le calme régnant dans ma maison.

J'espère que cette description vous a permis de plonger au cœur de ma rue. 
Comme pour les photographies, ces textes me servaient de support aux entretiens ultérieurs, avec lesquels ils entraient bien souvent en cohérence. Ils témoignaient ainsi avec finesse de différents rapports entretenus au quartier de résidence, parfois mieux décrits dans les récits des jeunes que dans leurs propos. L'ensemble des textes et photographies réalisés, couplé aux résultats des questionnaires, ont ensuite donné lieu à des opérations de valorisation, afin que les élèves puissent voir le résultat de leur travail. II leur était ainsi demandé, le plus souvent au début des projets, le mode de restitution de leur travail ayant leur préférence. Cette restitution a alors prise différentes formes: mise en place d'un blog, expositions dans les halls des établissements, au centre de documentation ou lors de journées portes ouvertes, réalisation d'un petit livret financé par le Conseil général de Seine Saint Denis...

A la suite de la réalisation des questionnaires, textes et photographies, des entretiens individuels d'une heure étaient proposés. II avait été bien expliqué que ces entretiens n'étaient pas obligatoires, bien qu'ils aient lieu le plus souvent durant les heures de cours et dans l'enceinte des établissements. La quasi-totalité des élèves ont accepté de se prêter au jeu, en raison sans doute de la bonne réception du projet dans son ensemble : la majorité des élèves se montra ainsi enthousiaste à l'égard d'activités qui sortaient du cadre scolaire habituel, y compris les élèves en difficulté et ayant un rapport compliqué à l'institution scolaire. Cela s'explique sans doute en partie par le fait que les mobilités ne soient pas un sujet trop intime, les élèves pouvant parler de ce thème entre eux. II n'est ainsi pas certain que de tels projets aient pu être menés sur le thème de la sexualité. Néanmoins, une minorité d'élèves sont restés en retrait, ne manifestant au contraire de leurs camarades aucun enthousiasme. II s'agissait principalement d'élèves introvertis ou avec une fréquentation très épisodique des établissements scolaires. Ces derniers ont certes accepté ensuite le principe d'un entretien, mais ils me confièrent ensuite que leur motivation principale était de manquer des heures de cours.

Je n'ai pas sollicité une autorisation des parents pour la réalisation de ces entretiens, pour les raisons exposées au début de cet article. L'absence d'autorisation me semblait d'autant moins problématique au niveau déontologique, que les entretiens étaient réalisés après que la réalisation et passation des questionnaires aient fait comprendre aux élèves ce que signifiait de participer à une enquête de sociologie. Le principe de l'enregistrement ayant été toujours accepté à trois exceptions près, une version sur CD était remise au jeune quelques jours après la réalisation de l'entretien, à la demande initiale d'une grande partie des élèves. J'ai également interrogé systématiquement les adolescents à la fin de l'entretien sur leur ressenti, la plupart me confiant à cette occasion leur satisfaction. Plusieurs d'entre eux me déclarèrent que cet entretien leur avait permis de mieux comprendre que la mobilité n'était pas innée et qu'ils avaient dû apprendre à se confronter à l'altérité. II s'agissait néanmoins essentiellement d'adolescents ayant des pratiques de mobilité spécifiques, très fortement tournées vers la flânerie urbaine. D'autres, plus rares, me confièrent leur impression d'avoir expérimenté une séance de "psy» leur ayant permis de mieux se connaître, confirmant ainsi que l'entretien avait permis un retour réflexif sur les pratiques. Enfin, une partie des élèves ayant peu l'occasion de se déplacer en dehors de leur quartier souligna que cela leur avait fait du bien de parler des problèmes de leur vie quotidienne à un intervenant extérieur. 
Ce questionnement immédiat sur leur ressenti n'était pas la seule occasion d'échange avec les élèves sur les entretiens. En effet, une fois les entretiens réalisés avec l'ensemble des volontaires, une séance de restitution était organisée en classe entière, suivie d'un débat sur les principaux résultats obtenus. Cette restitution était guidée à l'origine par des considérations déontologiques. Elle eut néanmoins des répercussions importantes au niveau des résultats scientifiques de ma recherche, en particulier sur la typologie des jeunes que j'avais effectuée en fonction de leurs pratiques de mobilité. Des jeunes, issus de différents établissements scolaires, sont venus ainsi me confier à la fin de la restitution qu'ils comprenaient ma typologie mais qu'ils se reconnaissaient en partie dans deux catégories. Ces remarques m'incitèrent à porter une attention plus soutenue aux processus d'apprentissage et de socialisation multiple des adolescents. Elles me permirent ainsi de complexifier mon approche initiale trop statique et rigoriste de la typologie

\section{Conclusion}

La méthode que je viens d'exposer d'une co-construction d'une recherche avec des adolescents possède donc des avantages éthiques et scientifiques. Elle était en tout cas adaptée à mon objet de recherche consistant à mieux comprendre les différentes épreuves auxquelles sont confrontés les adolescents de ZUS durant leurs mobilités. Elle ne prend sens qu'en complémentarité avec les autres matériaux ethnographiques et statistiques recueillis. Ces différentes méthodes s'éclairent mutuellement et soulèvent chacune des difficultés éthiques et scientifiques propres. J'ai cependant tiré deux enseignements majeurs de cette méthode relativement originale consistant à faire des adolescents des partenaires de recherche et non un simple objet d'étude. D'une part, les dimensions éthiques et scientifiques d'une recherche sur les adolescents ne sont guère dissociables, la méthode de collecte de données influant fortement sur le matériau recueilli. D'autre part, les adolescents sont sans nul doute compétents pour interpréter ce que la sociologie dit à leur propos. Ils ne sont ainsi pas les simples réceptacles d'une socialisation familiale ou dans l'apprentissage de normes comme on les présente souvent, mais également des acteurs capables et souvent désireux d'avoir un regard réflexif sur leurs pratiques.

\section{Bibliographie}

ALDERSON P. (1995) Listening to Children: Children, Ethics and Social Research, Londres, Barnardo's, 130p.

BECKER H. (2010) Comment parler de la société ? Artistes, écrivains, chercheurs et représentations sociales, Paris, La Découverte, 320p.

BELL N. (2008) " Ethics in child research: rights, reason and responsibilities ", Children's Geographies, 6/1, pp.7-20. 
BREVIGLIERI M. (2007) "Ouvrir le monde en personne. Une anthropologie des adolescences ", in Breviglieri M., Cicchelli V., Adolescences méditerranéennes. L'espace public à petit pas, Paris, L'Harmattan, pp.19-59.

DANIC I., DELALANDE J., RAYOU P. (2006), Enquêter auprès d'enfants et de jeunes. Objets, méthodes et terrains de recherche en sciences sociales, Rennes, PUR, 216p.

DUBET F., MARTUCELLI D. (1996) A l'école. Sociologie de l'expérience scolaire, Paris, Seuil.

Fine M., fReUdenberg N., PAYNe A., PERKINS T., SMITH K., WANZER, K. (2003) "Anything Can Happen with Police Around: Urban Youth Evaluate Strategies of Surveillance in Public Places», Journal of Social Issues, 59, pp.141-158.

FIRDION J-M., MARPSAT M., BOZON M. (1995), " Est-il légitime de mener des enquêtes statistiques auprès des sans-domicile ? Une question éthique et scientifique ", Revue française des affaires sociales, 2-3, pp.29-51.

GARFINKEL H. (1967) Studies in Ethnomethodology, Cambridge, Polity Press, 288p.

HART, R. (1992) Children's Participation: from tokenism to citizenship, Florence, UNICEF International Child Development Centre, $44 \mathrm{p}$.

KOKOREFF M. (1998) " Mobilités et polarisations des jeunes dans la ville ", in Haumont N. (dir.), L'urbain dans tous ses états. Faire, vivre et dire la ville, Paris, L'Harmattan, pp.245-254.

LEPOUTRE D. (2005) Souvenirs de familles immigrées, Paris, Odile Jacob, 377p.

MASSON J. (2004) "The legal context», in Fraser S. (dir.), Doing Research with Children and Young People, Londres, Sage, pp.43-58.

MORROW V. (2008) "Ethical dilemmas in research with children and young people about their social environments ", Children's Geographies, 6/1, pp.49-61.

OPPENCHAIM N. (2009) "Mobilités quotidiennes et ségrégation: le cas des adolescents de Zones Urbaines Sensibles franciliennes ", Espace populations sociétés, 2, pp.215-226.

OPPENCHAIM N. (2011) "Les adolescents de catégories populaires ont-ils des pratiques de mobilités quotidiennes spécifiques ? Le cas des zones urbaines sensibles franciliennes ", Recherche Transports Sécurité, 27/2, pp.93-103.

SIME D. (2008) "Ethical and methodological issues in engaging young people living in poverty with participatory research methods", Children's Geography, 6/1, pp.63-78.

SKELTON T. (2008) "Research with children and young people: exploring the tensions between ethics, competence and participation ", Children's Geographies, 6/1, pp.2136.

VASSY C., KELLER R. (2008) " Faut-il contrôler les aspects éthiques de la recherche en sciences sociales, et comment ? ", Mouvements, 55-56, pp.128-141. 
TONNELAT S., JOLE M., KORNBLUM W. (2007) "Vers une ethnographie participative ", in CEFAÏ D., SATURNO C. (dir), Isaac Joseph. Itinéraire d'un pragmatiste, Paris, Economica, pp.277-291.

ZAFFRAN J. (2010) Le temps de l'adolescence - Entre contrainte et liberté, Rennes, PUR. 